\title{
Note de synthèse - L'espérance de vie ajustée en fonction de l'état de santé au Canada : Rapport de 2012 présenté par l'Agence de la santé publique du Canada
}

\author{
Comité directeur sur l'espérance de vie ajustée en fonction de l'état de santé de l'Agence de la santé publique du \\ Canada (1)
}

L'espérance de vie ajustée en fonction de l'état de santé est un indicateur du nombre moyen d'années de vie en bonne santé que peut s'attendre à connaître une personne. Il s'agit d'une mesure agrégée qui englobe la durée de la vie et la qualité de la vie. Autrement dit, l'espérance de vie ajustée en fonction de l'état de santé combine l'expérience de mortalité et l'expérience de morbidité en une simple mesure agrégée de la santé de la population. La statistique ainsi obtenue peut être utile à l'évaluation du fardeau imposé par les maladies et les blessures, à l'étude des facteurs de risque au sein d'une population ainsi qu'à l'examen du rendement des efforts déployés dans le domaine de la santé publique.

Le présent rapport, intitulé L'espérance de vie ajustée en fonction de l'état de santé au Canada : Rapport de 2012 présenté par l'Agence de la santé publique du Canada ${ }^{1}$, fournit des estimations de l'espérance de vie ajustée en fonction de l'état de santé des Canadiens, selon que ces derniers soient atteints ou non de certaines maladies chroniques (diabète et cancer) ou de certains états chroniques (hypertension artérielle) et selon leur situation socioéconomique (revenu). Les estimations sont présentées pour les femmes et les hommes de même que pour différents groupes d'âge.

Il existe un lien entre une situation socioéconomique défavorable et la diminution de l'espérance de vie ajustée en fonction de l'état de santé. En 2001, au Canada, les femmes et les hommes se trouvant dans le tertile supérieur de revenu avaient, à la naissance, une espérance de vie ajustée en fonction de l'état de santé de 72,3 années et 70,5 années, respectivement. Par rapport aux membres de la catégorie de revenu la plus élevée, les personnes se trouvant dans le tertile inférieur de revenu affichaient une espérance de vie ajustée en fonction de l'état de santé moins élevée à la naissance, l'écart étant de 3,2 années pour les femmes et 4,7 années pour les hommes.

Les maladies et les états chroniques s'accompagnent d'une diminution notable de l'espérance de vie ajustée en fonction de l'état de santé. Les estimations de l'espérance de vie ajustée en fonction de l'état de santé selon la présence ou l'absence de maladies chroniques ont été établies, pour ce rapport, à partir de l'expérience de mortalité et de l'expérience de morbidité des personnes atteintes ou non de diabète et/ou d'hypertension artérielle (pour la période de 2004 à 2006) et des personnes atteintes ou non de cancer (pour la période de 2002 à 2005) Selon les résultats de l'étude, la cohorte de personnes atteintes de diabète à 55 ans connaît une diminution de l'espérance de vie ajustée en fonction de l'état de santé de 5,8 années pour les femmes et 5,3 années pour les hommes par rapport à la cohorte non atteintes de diabète. Pour sa part, la diminution estimée que connaît la cohorte de personnes atteintes d'hypertension artérielle à 55 ans s'établit à 2,0 années pour les femmes et 2,7 années pour les hommes. Enfin, la cohorte de personnes atteintes de cancer à 65 ans connaît une diminution de l'espérance de vie ajustée en fonction de l'état de santé de 10,3 années pour les femmes et 9,2 années pour les hommes.

Le présent rapport communique des renseignements qui s'avéreront utiles aux chercheurs, aux praticiens et aux responsables des politiques dans le domaine de la santé publique. À l'avenir, d'autres rapports pourront élargir la portée de l'analyse afin d'aborder l'espérance de vie ajustée en fonction de l'état de santé selon la présence ou l'absence de certains facteurs de risque comportementaux comme l'obésité, l'inactivité physique ou le tabagisme.

Le rapport complet est consultable en ligne à la page : http://www.phac-aspc. gc.ca/cd-mc/hale-evas-pdf-fra.php

\section{Références}

1. Comité directeur sur l'espérance de vie ajustée en fonction de l'état de santé de l'Agence de la santé publique du Canada. L'espérance de vie ajustée en fonction de l'état de santé au Canada : Rapport de 2012 présenté par l'Agence de la santé publique du Canada. Consultable en ligne à la page : http://www.phac-aspc.gc.ca/cd-mc/haleevas-pdf-fra.php

Rattachement de l'auteur :

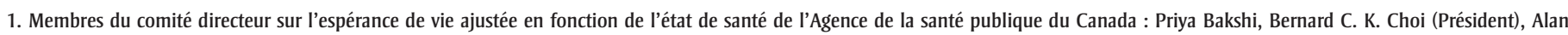

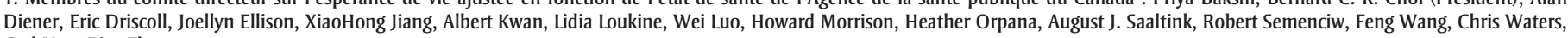
Carl Yue, Rita Zhang.

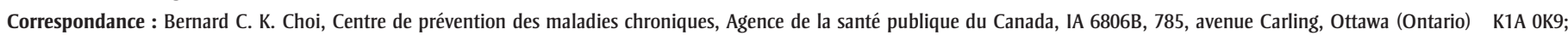
tél. : (613) 957-1074; courriel : Bernard.Choi@phac-aspc.gc.ca 